\title{
De Novo Detrusor Underactivity and Other Urodynamic Findings after Radical Prostatectomy: A Systematic Review
}

\author{
Maciej Oszczudłowski *, Konrad Bilski, Mieszko Kozikowski and Jakub Dobruch
}

check for updates

Citation: Oszczudłowski, M.; Bilski, K.; Kozikowski, M.; Dobruch, J. De Novo Detrusor Underactivity and Other Urodynamic Findings after Radical Prostatectomy: A Systematic Review. Medicina 2022, 58, 381. https://doi.org/10.3390/ medicina58030381

Academic Editors: Mikolaj Przydacz and Piotr Ludwik Chłosta

Received: 9 January 2022

Accepted: 1 March 2022

Published: 4 March 2022

Publisher's Note: MDPI stays neutral with regard to jurisdictional claims in published maps and institutional affiliations.

Copyright: (C) 2022 by the authors. Licensee MDPI, Basel, Switzerland. This article is an open access article distributed under the terms and conditions of the Creative Commons Attribution (CC BY) license (https:// creativecommons.org/licenses/by/ $4.0 /)$.

\author{
Urology Clinic, Centre of Postgraduate Medical Education, 01-813 Warsaw, Poland; \\ konradbilski@gmail.com (K.B.); mieszkokozikowski9@gmail.com (M.K.); jdobruch@cmkp.edu.pl (J.D.) \\ * Correspondence: moszczudlowski@cmkp.edu.pl
}

\begin{abstract}
Background and objectives: The aim of this systematic review is to evaluate the impact of radical prostatectomy $(\mathrm{RP})$ on bladder function, with special attention towards detrusor underactivity investigated with the means of urodynamic evaluation. Materials and Methods: The review was performed in accordance with the PRISMA statement and was registered in the PROSPERO (ID\#: CRD42020223480). The studied population was limited to men with prostate cancer who underwent urodynamic study prior to and after radical prostatectomy. Eight hundred twenty-seven studies were screened, with twenty-five finally included. A qualitative analysis was performed. Rates of detrusor underactivity (DU) before surgery were reported in eight studies and ranged from $1.6 \%$ to $75 \%$ (median of $40.8 \%$ ). DU occurred de novo after RP in $9.1 \%$ to $37 \%$ of patients (median of $29.1 \%$ ). On the other hand, preexisting DU resolved in $7 \%$ to $35.5 \%$ of affected men. Detrusor overactivity (DO) was the most frequently reported outcome, being assessed in 23 studies. The rate of DO preoperatively was from $5 \%$ to $76 \%$ (median of $25 \%$ ). De novo was reported in $2.3-54.4 \%$ of patients (median of $15 \%$ ) and resolved after RP in $19.6 \%$ to $87.5 \%$ (median of $33 \%$ ) of affected patients. Baseline rates of bladder outlet obstruction (BOO) varied between studies from $19 \%$ to $59.3 \%$, with a median of $27.8 \%$. The most pronounced change after surgery was the resolution of $\mathrm{BOO}$ in $88 \%$ to $93.8 \%$ (median of $92 \%$ ) of affected patients. Results: Rates of de novo impaired bladder compliance (IBC) varied from $3.2 \%$ to $41.3 \%$ (median of $13.3 \%$ ), whereas the resolution of IBC was reported with rates ranging from $0 \%$ to $47 \%$ (median of $4.8 \%$ ). Conclusions: $\mathrm{BOO}, \mathrm{DO}$, and DU are frequently diagnosed in men scheduled for RP. BOO is improved after RP in most patients; however, there is still a substantial rate of patients with de novo DU as well as DO which may impair functional outcomes and quality of life.
\end{abstract}

Keywords: radical prostatectomy; detrusor underactivity; urodynamic study; detrusor overactivity; bladder outlet obstruction; impaired bladder compliance

\section{Introduction}

Prostate cancer (PCa) is the second most common cancer in men worldwide, accounting for $15 \%$ of all diagnosed cancers [1]. Implementation of prostate-specific antigen testing and the extended life span of elderly men in developed countries led to a substantial increase in PCa incidence, followed by rising numbers of younger patients with long life expectancy subjected to radical prostatectomy (RP). Moreover, novel tools based on genetic alterations and epigenetic interactions are evaluated for the determination of indolent or aggressive tumour behaviour [2]. This phenomenon resulted in a growing interest in novel surgical techniques that would limit the functional morbidity of RP [3]. The impact of the surgery on men's well-being is well documented. The quality of life (Qol) of those who underwent radical prostatectomy is significantly worse when compared with their noncancer counterparts [4]. Among several domains of Qol, the urinary function has been extensively investigated, including urinary incontinence as one of the most bothersome lower urinary tract symptoms (LUTS). Although less frequently studied, other LUTS are not uncommon 
after RP [5]. Unfortunately, the high preoperative incidence of LUTS further complicates research on the impact of surgery on lower urinary tract (LUT) function afterwards [6-9].

Conditions that cause postprostatectomy urinary incontinence are well known [10-12], yet the number of other bladder function alterations that may influence the postsurgical urinary domain of quality of life (QOL) have not been thoroughly investigated. Among others, these apply to detrusor underactivity, detrusor overactivity, bladder outlet obstruction, and reduced bladder compliance. Although novel tools are evolving [13], the most precise method to evaluate them all is the urodynamic pressure-flow study (UDS). Therefore, the aim of our systematic review is to evaluate the impact of radical prostatectomy on bladder function, with special attention towards detrusor underactivity investigated with the means of urodynamic evaluation.

\section{Materials and Methods}

The conduct and reporting of this systematic review were performed in accordance with the guidelines of the Centre of Reviews and Dissemination as well as the Preferred Reporting Items for Systematic Reviews and Meta-Analyses (PRISMA) statement $[14,15]$. The review protocol was registered in the PROSPERO International prospective register of systematic reviews (ID\#: CRD42020223480). The studied population was limited to men with prostate cancer who underwent urodynamic study both prior to and at least once after radical prostatectomy. Studies without preplanned UDS before and after RP were excluded in accordance with the review protocol. Inclusion and exclusion criteria are summarised in Table 1.

Table 1. Eligibility criteria.

\begin{tabular}{lll}
\hline \multicolumn{1}{c}{ Inclusion Criteria } & \multicolumn{1}{c}{ Exclusion Criteria } \\
\hline (1) $\quad \begin{array}{l}\text { Studies on patients who underwent } \\
\text { radical prostatectomy for the treatment of } \\
\text { prostate cancer (open, laparoscopic } \\
\text { or robotic). }\end{array}$ & (1) & $\begin{array}{l}\text { Studies on patients who underwent } \\
\text { radiotherapy for prostate cancer prior to } \\
\text { surgery or during follow-up. }\end{array}$ \\
(2) $\begin{array}{l}\text { Studies on patients who underwent } \\
\text { urodynamic studies performed both } \\
\text { before and after radical prostatectomy. }\end{array}$ & $\begin{array}{l}\text { Studies on a predefined subgroup of } \\
\text { patients undergoing radical } \\
\text { prostatectomy, such as patients with } \\
\text { neurogenic bladder, determined lower } \\
\text { urinary tract symptoms, persistent } \\
\text { postprostatectomy stress } \\
\text { (3) } \begin{array}{l}\text { Full text published studies; articles in } \\
\text { English only. }\end{array}\end{array}$ & \\
&
\end{tabular}

The main outcomes appraised in this study are the incidence of detrusor underactivity (DU) that occurred after RP (de novo) as well as the total rate of DU in patients after RP. Additionally, the incidence of de novo and total detrusor overactivity (DO), impaired bladder compliance (IBC), and bladder outlet obstruction (BOO) after RP were assessed.

Electronic literature searches were conducted on 10 November 2020 using Pubmed, Cochrane Library, Web of Science, Scopus, and Embase databases. The search was then rerun on 10 December 2021, prior to the final analysis. The staged process of studies selection is illustrated in Figure 1, while detailed searching strategies for each database are listed in Supplementary Materials (Table S1). Screening, assessment against the predetermined eligibility criteria, and risk of bias assessments (National Heart, Lung, and Blood Institute Study Quality Assessment Tools) were performed by two researchers independently (M.O., K.B.). An online, cloud-based tool (http://rayyan.qcri.org, accessed on 12 November 2020) was used for the screening and eligibility assessment process [16]. Researchers were blinded to each other's results, whereas discordant decisions were resolved by consensus with the assistance of an additional independent researcher (M.K.). Data were collected into electronic data extraction form, which was first piloted and then applied by two independent investigators (M.O., K.B.). Collected data includes study characteristics, demographic data, characteristics of the intervention, baseline and follow-up urodynamic data, and patient-reported outcome measures (PROMs). 


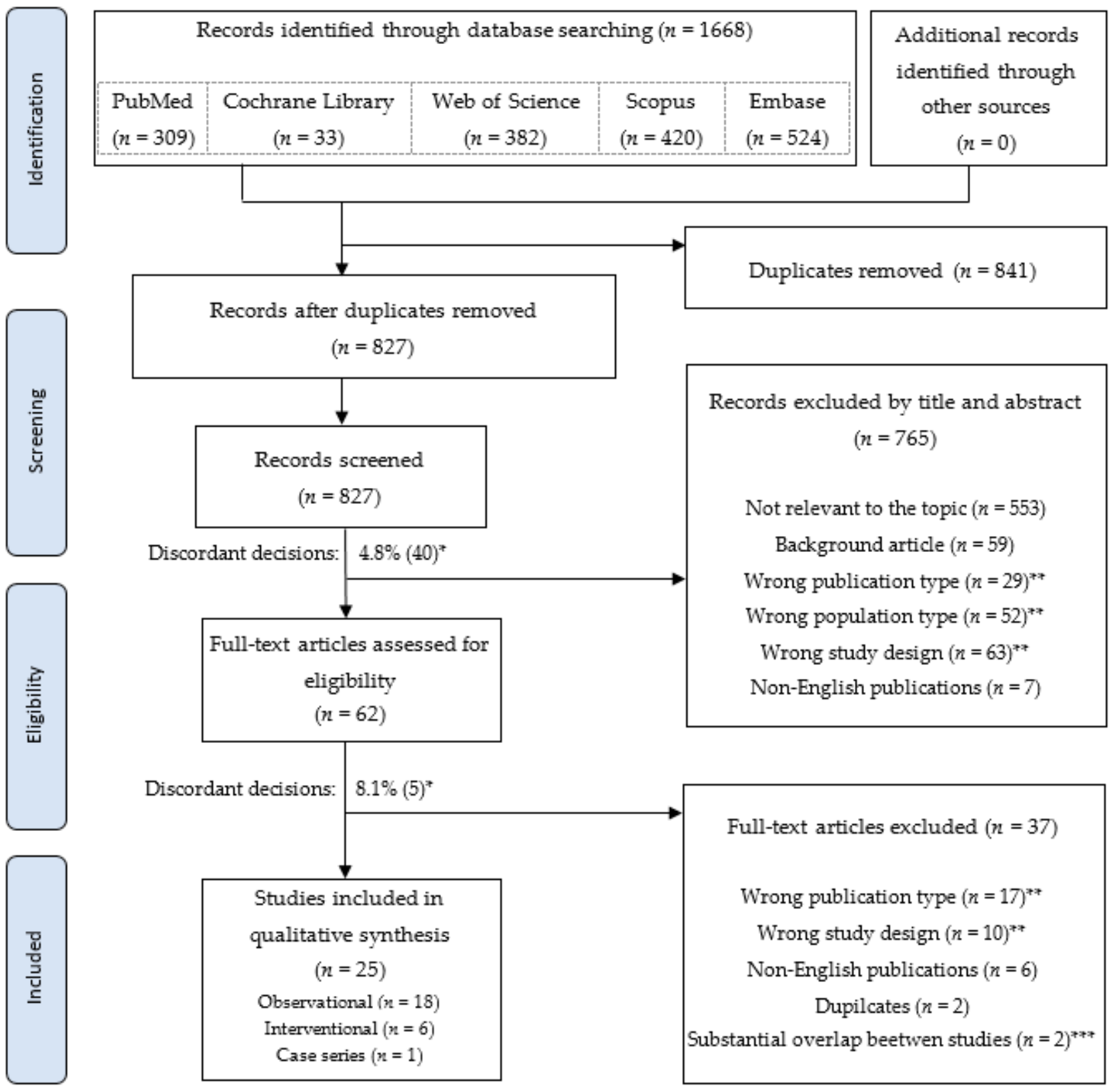

Figure 1. PRISMA diagram. The flow of records through the searching and appraisal process. * percent (number) of discordant decisions between investigators, resolved by consensus. ** according to the criteria presented in Table $1 .{ }^{* * *}$ based on additional data obtained from the author.

\section{Results}

A total of 827 studies were screened, 62 identified as relevant and 27 initially qualified for synthesis [17-43] (Figure 1). Populations from three [26-28] of the last-mentioned studies were, however, substantially overlapped $(72 \%$ to $90 \%$, based on data obtained from the author), which led to further exclusions [27,28]. Finally, 25 studies were included in the qualitative synthesis, with 16 of them being prospective studies. Four of included twenty-five articles came from two authors, indicating some risk of population overlap $[19,20,32,33]$, despite differences in years of inclusion, number of patients and populations characteristic. Attempt to contact authors for clarification was ineffective in these cases.

Included articles vary substantially in terms of length of follow up, type of intervention, and definitions of reported outcomes. Considering this heterogeneity, quantitative synthesis was not performed. 


\subsection{Baseline Characteristics}

The total number of patients in included studies was 1387 (age range 13-110). In the case of 47 patients, no adequate data was reported [24,30,31]. Finally, the number of patients included in qualitative synthesis was 1340 (age range 13-110) (data presented in Table 2), with a mean age range from 61.9 to 72.1 years (SD 2.6). At least one UDS after RP was performed in 1298 from 1340 patients (97\%). The total of 42 from 1340 patients had no UDS postoperatively (Table S2) $[22,25,35,36,38]$. Patients in thirteen studies were treated with open radical prostatectomy (ORP) [17-21,25,30,31,35,37-39,41]. Two studies reported outcomes after laparoscopic radical prostatectomy (LRP) [22,32] whereas five studies after robot-assisted laparoscopic radical prostatectomy (RALP) $[23,26,40,42,43]$. Four studies included a mixed population of patients treated either with ORP or LRP $[24,33,34,36]$. In one study, either LRP or RALP was performed [24]. Application of nerve-sparing technique was reported in 15 studies, ranging from $0 \%$ to $100 \%$ in treated patients $[33,40]$. The presence and range of lymphadenectomy performed during surgery were not reported in the majority of articles. Urodynamic evaluation in most reports adheres to rules outlined in the Good Urodynamic Practice statement [44]. Baseline demographics of included studies are collected in Table 2.

\subsection{Postoperative Reporting}

The length of follow-up ranged from 1 to 36 months [39,42], with the first assessment being performed from 3-4 days following catheter removal to 12 months after surgery $[29,42]$. Status of urinary continence was the most often reported functional outcome after RP (in 19 studies), although based on different timespans and assessment tools. Incontinence rates ranged from $0 \%$ to $98 \%$.

\subsubsection{Detrusor Contractility}

Detrusor contractility was assessed in 10 studies embracing in total 603 patients (Table 2) [18-20,26,29,32,34-36,42]. Definitions of detrusor underactivity (DU) and mathematical tools used for its assessment varied, however, substantially between studies (Table 3). Rates of impaired detrusor contractility before surgery were reported in eight studies with a median of $40.8 \%(1.6 \%$ to $75 \%)$ [18,42]. In one study, baseline detrusor contractility was not evaluated at all [32], whereas in another, bladder contractility was characterised as a continuous variable [29]. Absolute rates of DU initially after RP increased in five studies $[19,20,26,35,36]$ with range from $13.4 \%$ to $42 \%$, however in only two, these changes were significant $(18.4 \%$ and $20.5 \%, p<0.01)$ [19,20]. Furthermore, both reports came from the same author, indicating some risk of patients overlap. In three of the five last-mentioned studies, rates of DU insignificantly decreased afterwards with a range from $11.1 \%$ to $34.3 \%[19,20,26]$. In the other two studies, DU was assessed only once after surgery (6.5 and 12 months, respectively), and no significant changes of DU were revealed [18,34]. In one study, bladder contractility increased in the term of contractility pattern [29].

Rates of de novo DU after RP were reported in eight studies [19,20,26,32,34-36,42] with a median of $29.1 \%(9.1 \%$ to $37 \%)$ on the first follow-up. In the case of three of those studies, further follow-up assessment was conducted, revealing de novo DU in $6 \%$ to $25 \%$ of evaluated patients. On the other hand, preexisting DU resolved in $7 \%$ to $35.5 \%$ of the studied population. PROMs were reported in four [29,34,36,42] of the ten studies assessing DU, with IPSS and its QOL domain being most often used (Table 3). Only in the study by Natsume et al. [36] the mean IPSS number significantly increased, whereas, in others, differences were insignificant.

The coincidence of DU and other urodynamic alterations was not uncommon (Table 3) and was reported in five studies [19,20,26,32,35]. The concomitance of DU and ISD (Intrinsic Sphincter Deficiency) was found to be the most frequent combination (range from $6 \%$ to $60 \%$ ). The second most common one was the mixture of DU and DO, with a median of $29.6 \%$ (6\% to $59 \%)$. 
Table 2. Qualitative synthesis of data from included studies.

\begin{tabular}{|c|c|c|c|c|c|c|c|c|c|c|c|c|c|}
\hline Year & Author & $\mathbf{N}$ & $\begin{array}{c}\text { F-UP } \\
\text { (Months) } \\
\text { (Range) }\end{array}$ & $\begin{array}{c}\text { Age (Years) } \\
\text { Mean } \\
\text { (Range) }\end{array}$ & $\begin{array}{c}\text { Type of } \\
\text { RP }\end{array}$ & NS & UI & $\begin{array}{c}\text { PVR }(\mathrm{mL}) \\
\Delta \text { PVR }\end{array}$ & $\begin{array}{c}\text { Bcap. }(\mathrm{mL}) \\
\Delta \text { Bcap. } \\
(\mathrm{mL})\end{array}$ & $\begin{array}{c}\mathrm{BC} \\
\left.\text { (mL/cmH } \mathrm{m}_{2} \mathrm{O}\right) \\
\text { or/and IBC } \\
\Delta \mathrm{BC} ; \Delta \mathrm{IBC}\end{array}$ & $\begin{array}{c}\text { BOO } \\
\Delta \text { BOO }\end{array}$ & $\begin{array}{c}\text { DO } \\
\Delta \text { DO }\end{array}$ & $\begin{array}{c}\mathrm{DU} \\
\Delta \mathrm{DU}\end{array}$ \\
\hline \multirow[t]{2}{*}{1984} & Rudy & 17 & Pre-op. & 64.2 & ORP & - & $19 \%(3 / 16)$ & - & 365 & - & - & $25 \%(4 / 16)$ & - \\
\hline & & 14 & 6 & & & & $\begin{array}{c}87 \% \\
(14 / 16)\end{array}$ & - & $\begin{array}{c}293 \\
\downarrow 72+\end{array}$ & - & - & $\begin{array}{c}14.3 \%(2 / 14) \\
\downarrow 10.7 \% \\
\text { R } 50 \%(2 / 4)\end{array}$ & - \\
\hline \multirow[t]{2}{*}{1986} & Hellstrom & 19 & Pre-op. & $63(49-75)$ & ORP & - & $\begin{array}{c}5.3 \% \\
(1 / 19) \\
\end{array}$ & $30 \pm 10.3$ & $550 \pm 34.3$ & $37 \pm 10.40$ & - & $5.3 \%(1 / 19)$ & - \\
\hline & & 19 & 6 & & & & $\begin{array}{c}32 \% \\
(16 / 19) \\
\end{array}$ & $\begin{array}{c}0 \pm 4.68 \\
\downarrow 30 *\end{array}$ & $\begin{array}{c}450 \pm 35.1 \\
\downarrow 100 \dagger\end{array}$ & $\begin{array}{c}23 \pm 3.16 \\
\downarrow 14^{*}\end{array}$ & - & $5.3 \%(1 / 19)$ & - \\
\hline \multirow[t]{2}{*}{1992} & Constantin & 13 & & $62 \pm 1.7$ & ORP & $\begin{array}{l}61.5 \% \\
(8 / 13) \\
\end{array}$ & - & $150 \pm 37$ & $494 \pm 42$ & 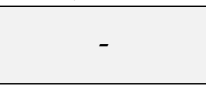 & - & $\begin{array}{c}76.9 \% \\
(10 / 13) \\
\end{array}$ & - \\
\hline & & 13 & $22.9 \S \pm 1.1$ & $62 \pm 1.7$ & & & $\begin{array}{l}38.5 \% \\
(5 / 13)\end{array}$ & $\begin{array}{c}62 \pm 43 \\
\downarrow 88 \pm 32 \dagger\end{array}$ & $\begin{array}{c}469 \pm 55 \\
\quad \downarrow 25\end{array}$ & - & - & $\begin{array}{c}61.5 \%(8 / 13) \\
\downarrow 15.4 \% \\
\text { N 8\% (1/13) } \\
\text { R 23\% (3/10) }\end{array}$ & - \\
\hline 1995 & Connoly & 17 & Pre-op. & - & ORP & - & $0 \%$ & 54.3 & $441.9^{a}$ & - & - & - & - \\
\hline \multirow[t]{2}{*}{1999} & Kleinhousє & 44 & Pre-op. & 68 & ORP & - & $0 \%$ & $38.1 \pm 90.6$ & $\begin{array}{c}375.4 \pm \\
171.9 \\
\end{array}$ & - & - & $\begin{array}{c}36.4 \% \\
(16 / 44) \\
\end{array}$ & - \\
\hline & & 44 & $7.8 \S(3-10)$ & & & & $\begin{array}{l}15.9 \% \\
(7 / 44)\end{array}$ & $\begin{array}{c}5.4 \pm 10.6 \\
\downarrow 32.7 \dagger\end{array}$ & $\begin{array}{c}427.8 \pm \\
144.7 \\
\uparrow 52.4 *\end{array}$ & - & - & $\begin{array}{c}6.8 \%(3 / 44) \\
\downarrow 29.6 \% \\
\text { N } 2.3 \% 1 / 44 \\
\text { R 87.5\% } \\
(\mathbf{1 4 / 1 6 )} \\
\end{array}$ & - \\
\hline \multirow[t]{3}{*}{2000} & John & 39 & Pre-op. & - & ORP & $\begin{array}{l}20.5 \% \\
(8 / 39) \\
\end{array}$ & $0 \%$ & - & $483 \pm 168$ & $49 \pm 35$ & - & - & - \\
\hline & & 34 & 1.5 & & & & $\begin{array}{c}82.4 \% \\
(28 / 34) \\
\end{array}$ & - & $376.8^{a}$ & $18.71^{\mathrm{a}}$ & - & - & - \\
\hline & & 34 & 6 & & & & $\begin{array}{l}17.6 \% \\
(6 / 34)\end{array}$ & - & $\begin{array}{l}430.7^{\mathrm{a}} \\
\downarrow 53.9\end{array}$ & $\begin{array}{c}21.18^{\mathrm{a}} \\
\uparrow 2.5\end{array}$ & - & - & - \\
\hline
\end{tabular}


Table 2. Cont.

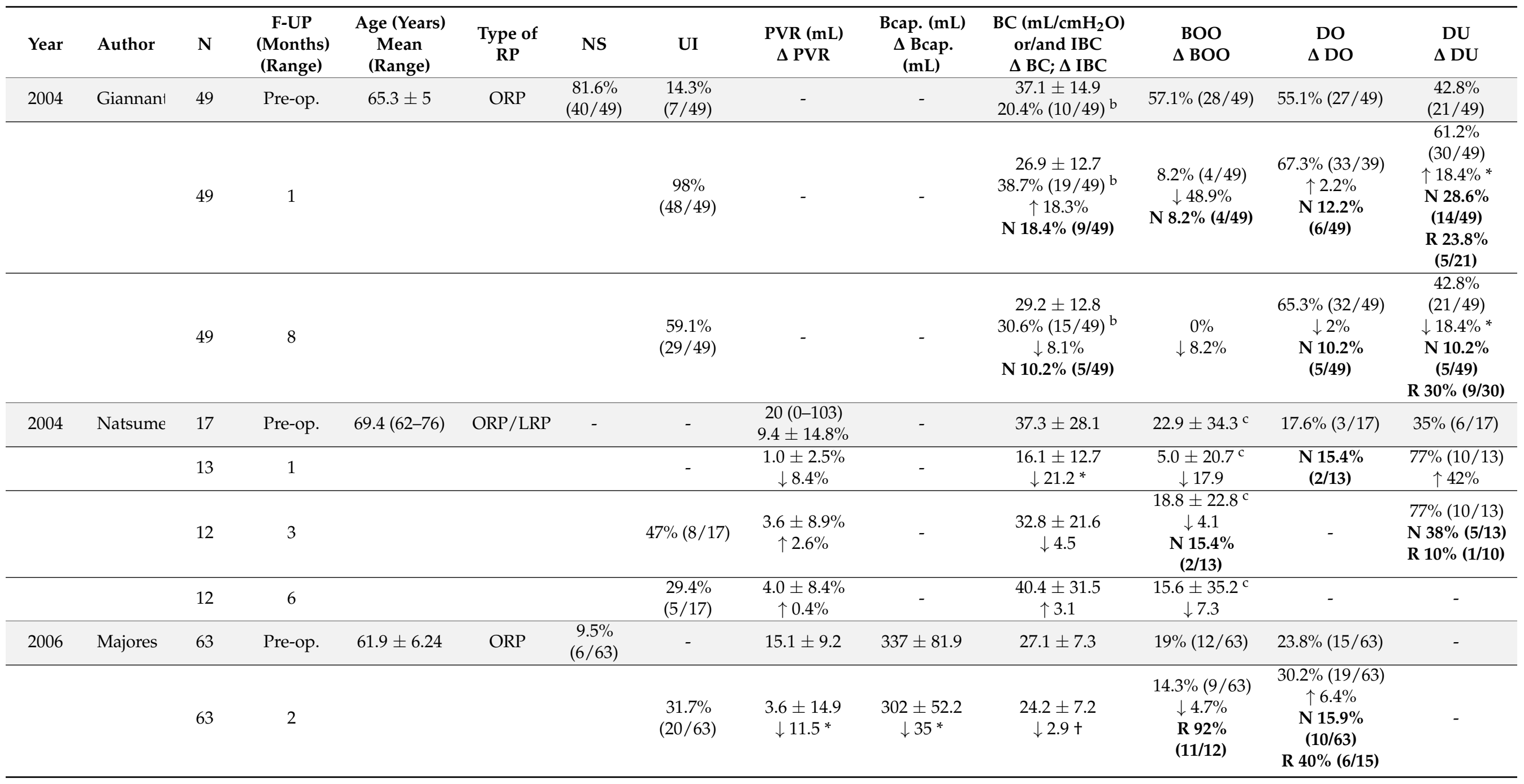


Table 2. Cont.

\begin{tabular}{|c|c|c|c|c|c|c|c|c|c|c|c|c|c|}
\hline Year & Author & $\mathbf{N}$ & $\begin{array}{c}\text { F-UP } \\
\text { (Months) } \\
\text { (Range) } \\
\end{array}$ & $\begin{array}{c}\text { Age (Years) } \\
\text { Mean } \\
\text { (Range) } \\
\end{array}$ & $\begin{array}{c}\text { Type of } \\
\text { RP }\end{array}$ & NS & UI & $\begin{array}{c}\text { PVR }(\mathrm{mL}) \\
\Delta \text { PVR }\end{array}$ & $\begin{array}{c}\text { Bcap. }(\mathrm{mL}) \\
\Delta \text { Bcap. } \\
(\mathrm{mL})\end{array}$ & $\begin{array}{c}\mathrm{BC}\left(\mathrm{mL} / \mathrm{cmH}_{2} \mathrm{O}\right) \\
\text { or/and IBC } \\
\Delta \mathrm{BC} ; \Delta \mathrm{IBC} \\
\end{array}$ & $\begin{array}{c}\text { BOO } \\
\Delta \text { BOO }\end{array}$ & $\begin{array}{c}\text { DO } \\
\Delta \text { DO }\end{array}$ & $\begin{array}{c}\mathrm{DU} \\
\Delta \mathrm{DU}\end{array}$ \\
\hline \multirow[t]{4}{*}{2006} & Noguchi & 45 & Pre-op. & $68 \pm 4.8$ & ORP & - & - & - & $381.3^{\mathrm{a}}$ & - & - & - & - \\
\hline & & 45 & 0.25 & & & & $50.9 \%{ }^{a}$ & - & $\begin{array}{l}240.9^{\mathrm{a}} \\
\downarrow 140.4\end{array}$ & - & - & $15.3 \%{ }^{a}$ & - \\
\hline & & 45 & 1 & & & & $33.2 \%{ }^{a}$ & - & $\begin{array}{l}283.6^{\mathrm{a}} \\
\downarrow 97.7\end{array}$ & - & - & $\begin{array}{l}7.5 \%^{\mathrm{a}} \\
\downarrow 7.8 \%\end{array}$ & - \\
\hline & & 45 & 3 & & & & $19.9 \%^{a}$ & - & $\begin{array}{l}326.1^{\mathrm{a}} \\
\downarrow 55.2\end{array}$ & - & - & $\begin{array}{l}5.3 \%^{\mathrm{a}} \\
\downarrow 2.2 \%\end{array}$ & - \\
\hline \multirow[t]{2}{*}{2008} & Giannants & 54 & Pre-op. & $67 \pm 5$ & ORP & $\begin{array}{c}81.5 \% \\
(44 / 54)\end{array}$ & $0 \%$ & - & $\mathrm{N} / \mathrm{A}$ & $\begin{array}{c}38.3 \pm 12 \\
37.1 \%(20 / 54) b\end{array}$ & $\begin{array}{c}59.3 \% \\
(32 / 54)\end{array}$ & $\begin{array}{c}61.2 \% \\
(33 / 54)\end{array}$ & $\begin{array}{c}38.8 \% \\
(21 / 54) \\
\end{array}$ \\
\hline & & 54 & 8 & & & & $\begin{array}{c}70 \% \\
(40 / 54) *\end{array}$ & - & - & $\begin{array}{c}24.2 \pm 9.9 \\
\downarrow 14.1+ \\
53.7 \%(29 / 54) b \\
\uparrow 20.6 \% * \\
\text { N } 20.4 \%(\mathbf{1 1} / 54) \\
\text { R } 10 \%(\mathbf{2} / \mathbf{2 0})\end{array}$ & $\begin{array}{c}7.4 \%(4 / 54) \\
\downarrow 51.9 \% * \\
\text { N 7.4\% } \\
\mathbf{( 4 / 5 4 )} \\
\text { R 93.8\% } \\
\mathbf{( 3 0 / 3 2 )}\end{array}$ & $\begin{array}{c}70 \%(38 / 54) \\
\uparrow 8.8 \%+ \\
\mathbf{N} \mathbf{1 4 . 8 \%} \\
\mathbf{( 8 / 5 4 )} \\
\text { R 9.1\% } \\
\mathbf{( 3 / 3 3 )}\end{array}$ & $\begin{array}{c}59.3 \% \\
(32 / 54) \\
\uparrow 20.5 \% \text { * } \\
\text { N 29.6\% } \\
\text { (16/54) } \\
\text { R 23.8\% } \\
(\mathbf{5} / 21)\end{array}$ \\
\hline \multirow[t]{4}{*}{2009} & Matsukan & 63 & Pre-op. & $66.5(53-75)$ & LRP & $\begin{array}{c}19 \% \\
(12 / 63)\end{array}$ & - & - & $253.6(64.8)$ & $63.9(31.8)$ & - & - & - \\
\hline & & 63 & $4.3 \S(3-9)$ & & & & $\begin{array}{c}17.5 \% \\
(11 / 63)\end{array}$ & - & $240.5(60.9)$ & $\begin{array}{c}32.8(14.8) \\
\downarrow 31.1^{*}\end{array}$ & - & $12.3 \%^{\mathrm{a}}$ & - \\
\hline & & 58 & Pre-op. & $67(55-73)$ & ORP & $0 \%$ & - & - & - & - & - & - & - \\
\hline & & 58 & $6.1 \S(3-12)$ & & & & $\begin{array}{c}22.4 \% \\
(13 / 58)\end{array}$ & - & $206^{a}$ & 21.48 & - & $42.6 \%{ }^{a}$ & - \\
\hline
\end{tabular}


Table 2. Cont.

\begin{tabular}{|c|c|c|c|c|c|c|c|c|c|c|c|c|c|}
\hline Year & Author & $\mathbf{N}$ & $\begin{array}{c}\text { F-UP } \\
\text { (Months) } \\
\text { (Range) }\end{array}$ & $\begin{array}{c}\text { Age (Years) } \\
\text { Mean } \\
\text { (Range) }\end{array}$ & $\begin{array}{c}\text { Type of } \\
\text { RP }\end{array}$ & NS & UI & $\begin{array}{c}\text { PVR (mL) } \\
\Delta \text { PVR }\end{array}$ & $\begin{array}{c}\text { Bcap. }(\mathrm{mL}) \\
\Delta \text { Bcap. }(\mathrm{mL})\end{array}$ & $\begin{array}{c}\mathrm{BC} \\
\left(\mathrm{mL} / \mathrm{cmH}_{2} \mathrm{O}\right) \\
\text { or/and IBC } \\
\Delta \mathrm{BC} ; \Delta \mathrm{IBC}\end{array}$ & $\begin{array}{c}\text { BOO } \\
\Delta \text { BOO }\end{array}$ & $\begin{array}{c}\text { DO } \\
\Delta \text { DO }\end{array}$ & $\begin{array}{c}\text { DU } \\
\Delta \text { DU }\end{array}$ \\
\hline \multirow[t]{2}{*}{2010} & Matsuka' & 110 & Pre-op. & $66.1^{\mathrm{a}}$ & LRP & - & - & - & $255^{a}$ & - & - & $25.5 \%(28 / 110)$ & - \\
\hline & & 110 & $3.8 \S(2-5)$ & & & & - & - & $248.7^{\mathrm{a}}$ & - & - & $\begin{array}{c}32.7 \%(36 / 110) \\
\uparrow 7.2 \% \\
\text { N } 20.9 \% \\
(\mathbf{2 3 / 1 1 0 )} \\
\text { R } 53.6 \%(\mathbf{1 5} / \mathbf{2 8 )})\end{array}$ & $\begin{array}{l}\text { N 9.1\% } \\
(10 / 110)\end{array}$ \\
\hline \multirow[t]{3}{*}{2010} & Song & 72 & Pre-op. & $64(49-77)$ & ORP & - & - & $14.5 \pm 18.2 \%$ & $393 \pm 91.5$ & $2.8 \%(2 / 72)^{b}$ & $\begin{array}{c}20.8 \% \\
(15 / 72)\end{array}$ & $38 \%(27 / 72)$ & $4.2 \%(3 / 72)$ \\
\hline & & 72 & 3 & & & & $\begin{array}{c}46 \% \\
(33 / 72)\end{array}$ & - & 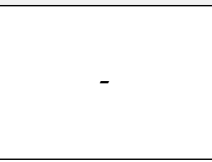 & - & $\begin{array}{c}5.6 \% \\
(4 / 72) \\
\downarrow 15.2 \%\end{array}$ & $\begin{array}{c}45.8 \% \\
\uparrow 7.8 \% \\
\mathbf{N} 16.7 \% \\
\mathbf{( 1 2 / 7 2 )}\end{array}$ & - \\
\hline & & 72 & 6 & & & & $\begin{array}{c}18 \% \\
(13 / 72)\end{array}$ & - & - & - & $\begin{array}{c}2.8 \% \\
(2 / 72) \\
(\downarrow) 2.8 \%\end{array}$ & N $1.4 \%(1 / 72)$ & - \\
\hline \multirow[t]{2}{*}{2012} & Mitsui & 43 & Pre-op. & $65^{a}$ & ORP/LRP & - & $0 \%$ & $60.2 \pm 86.3$ & $379 \pm 148$ & $0 \% \mathrm{~b}$ & $\begin{array}{c}30.2 \% \\
(13 / 43)\end{array}$ & $11.6 \%(5 / 43)$ & $48.8 \%(21 / 43)$ \\
\hline & & 43 & 12 & & & & - & $\begin{array}{c}22.7 \pm 65.8 \\
\downarrow 337.5^{*}\end{array}$ & $320 \pm 112$ & $0 \% \mathrm{~b}$ & $\begin{array}{l}7 \%(3 / 43) \\
\downarrow 23.2 \% *\end{array}$ & $\begin{array}{c}9.3 \%(4 / 43) \\
\downarrow 2.3 \% \\
\text { N } 9.3 \%(4 / 43) \\
\text { R } 60 \%(3 / 5)\end{array}$ & $\begin{array}{c}44.2 \%(19 / 43) \\
\downarrow 4.6 \%+ \\
\text { N } 9.3 \%(4 / 43) \\
\text { R } 28.6 \%(6 / 21)\end{array}$ \\
\hline \multirow[t]{2}{*}{2012} & Dubelma & 66 & Pre-op. & $64(60-67) \ddagger$ & ORP & $\begin{array}{l}56.1 \% \\
(37 / 66)\end{array}$ & - & & 473 & $12 \%(8 / 65)^{b}$ & $\begin{array}{c}49.2 \% \\
(31 / 63)\end{array}$ & $26 \%(17 / 66)$ & $75 \%(48 / 64)$ \\
\hline & & 66 & 6.5 & & & & $\begin{array}{c}28.9 \% \\
(19 / 66)\end{array}$ & & $\begin{array}{c}435 \\
\downarrow 38+\end{array}$ & $18 \%(12 / 65)^{b}$ & $\begin{array}{c}28.6 \% \\
(18 / 63) \\
\downarrow 20.6 \% *\end{array}$ & $\begin{array}{c}21 \%(14 / 66) \\
\downarrow 5 \%\end{array}$ & $\begin{array}{c}70.3 \%(45 / 64) \\
\downarrow 4.7 \%+\end{array}$ \\
\hline
\end{tabular}


Table 2. Cont.

\begin{tabular}{|c|c|c|c|c|c|c|c|c|c|c|c|c|c|}
\hline Year & Author & $\mathbf{N}$ & $\begin{array}{c}\text { F-UP } \\
\text { (Months) } \\
\text { (Range) }\end{array}$ & $\begin{array}{c}\text { Age (Years) } \\
\text { Mean } \\
\text { (Range) }\end{array}$ & $\begin{array}{c}\text { Type of } \\
\text { RP }\end{array}$ & NS & UI & $\begin{array}{c}\text { PVR }(\mathrm{mL}) \\
\Delta \text { PVR }\end{array}$ & $\begin{array}{c}\text { Bcap. }(\mathrm{mL}) \\
\Delta \text { Bcap. }(\mathrm{mL})\end{array}$ & $\begin{array}{c}\mathrm{BC} \\
\left(\mathrm{mL} / \mathrm{cmH}_{2} \mathrm{O}\right) \\
\text { or/and IBC } \\
\Delta \mathrm{BC} ; \Delta \text { IBC }\end{array}$ & $\begin{array}{c}\text { BOO } \\
\Delta \text { BOO }\end{array}$ & $\begin{array}{c}\text { DO } \\
\Delta \text { DO }\end{array}$ & $\begin{array}{c}\text { DU } \\
\Delta \mathrm{DU}\end{array}$ \\
\hline \multirow[t]{2}{*}{2013} & Mucciarc & 100 & Pre-op. & $\begin{array}{c}65.6 \pm 5.4 \\
(50-77)\end{array}$ & ORP & - & - & - & - & $16 \%(16 / 100)^{b}$ & - & $12 \%(12 / 100)$ & $73 \%(73 / 100)$ \\
\hline & & 88 & 12 & & & - & - & - & - & - & N $9.3 \%(4 / 43)$ & $\begin{array}{c}68.2 \%(60 / 88) \\
\uparrow 56.2 \% \\
\text { N } 54.5 \% \\
(48 / 88)\end{array}$ & $\begin{array}{c}86.4 \% ;(76 / 88) \\
\uparrow 13.4 \% \\
\text { N } 31.8 \%(28 / 88)\end{array}$ \\
\hline \multirow[t]{3}{*}{2015} & Kadono & 63 & Pre-op. & $65.3 \pm 4.8$ & RALP & $\begin{array}{c}58.7 \% \\
(37 / 63)\end{array}$ & $0 \%$ & & $335.9 \pm 92.3$ & $\begin{array}{c}28.3 \pm 18.3 \\
33 \%(21 / 63)^{b}\end{array}$ & $25.4 \%(16 / 63)$ & $28.6 \%(18 / 63)$ & $22 \%(14 / 63)$ \\
\hline & & 63 & Post-op. & & & & $\begin{array}{c}84.1 \% \\
(53 / 63)\end{array}$ & - & $251.4 \pm 69.8$ & $\begin{array}{c}16.3 \pm 10.8 \\
\downarrow 12 * \\
73 \%(46 / 63) \\
\uparrow 40 \% * \\
\text { N } 41.3 \%(\mathbf{2 6 / 6 3 )} \\
\text { R } 4.8 \%(\mathbf{1} / \mathbf{2 1})\end{array}$ & $\begin{array}{c}6.3 \%(4 / 63) \\
\downarrow 19.1 \\
\text { N 3.2\% (2/63) } \\
\text { R 88\% (14/16) }\end{array}$ & $\begin{array}{c}22.2 \%(14 / 63) \\
\downarrow 6.4 \\
\text { N 3.2\% }(2 / 63) \\
\text { R 33.3\% (6/18) }\end{array}$ & $\begin{array}{c}49.2 \%(31 / 63) \\
\uparrow 27.2 \% \\
\text { N } 28.6 \%(\mathbf{1 8 / 6 3 )} \\
\text { R 7.1\% (1/14) }\end{array}$ \\
\hline & & 63 & 12 & & & & $\begin{array}{c}11 \% \\
(7 / 63)\end{array}$ & - & $338.1 \pm 91.5$ & $\begin{array}{c}27.1 \pm 21.7 \\
\downarrow 10.8^{*} \\
41.2 \%(26 / 63) b \\
\downarrow 31.8 \% * \\
\text { N } 3.2 \%(2 / 63) \\
\text { R } 47.8 \%(22 / 46)\end{array}$ & $\begin{array}{c}3.2 \%(2 / 63) \\
\downarrow 3.1 \% \\
\text { N } 15.9 \%(1 / 63) \\
\text { R 75\% }(3 / 4)\end{array}$ & $\begin{array}{c}22.2 \%(14 / 63) \\
\text { N } 9.5 \%(6 / 63) \\
\text { R 33.3\% (6/18) }\end{array}$ & $\begin{array}{c}38.1 \%(24 / 63) \\
\downarrow 11.1 \% \\
\text { N } 6.3 \%(4 / 63) \\
\text { R } 35.5 \%(\mathbf{1 1} / 31)\end{array}$ \\
\hline \multirow[t]{3}{*}{2017} & Jiang & 46 & Pre-op. & $69.2 \pm 7.9$ & LRP/RALI & $\begin{array}{c}34.8 \% \\
(23 / 66)\end{array}$ & - & $31.6 \pm 60.8$ & $304.0 \pm 131.7$ & $68.1 \pm 73.6$ & $25.2 \pm 33.7$ & $56.5 \%(26 / 46)$ & - \\
\hline & & 46 & $3-6$ & & & & - & $\downarrow 9.8 \pm 60.0$ & - & $\uparrow 6.6 \pm 108.3$ & $\downarrow 23.9 \pm 37.4^{*, \mathrm{c}}$ & $\begin{array}{c}52.2 \%(24 / 46) \\
\downarrow 4.3 \% \\
\text { N } 15.2 \%(7 / 46) \\
\text { R } 19.6 \%(9 / 46)\end{array}$ & - \\
\hline & & 46 & 12 & & & & - & $\downarrow 5.2 \pm 29.5$ & - & $\downarrow 37.5 \pm 112.9$ & $\downarrow 34.1 \pm 40.0^{*, \mathrm{c}}$ & $52.2 \%(24 / 46)$ & - \\
\hline \multirow[t]{2}{*}{2017} & Kitta & 37 & Pre-op. & $65(53-74)$ & ORP/LRP & $\begin{array}{c}46 \% \\
(17 / 37)\end{array}$ & - & $48.6 \pm 66.1$ & $388 \pm 139$ & - & $24,2 \%(9 / 37)$ & $10.8 \%(4 / 37)$ & $\begin{array}{c}114.6 \pm 35.6^{\mathrm{d}} \\
-0.4 \pm 2.0^{\mathrm{e}}\end{array}$ \\
\hline & & 37 & 12 & & & & - & $\begin{array}{c}10.1 \pm 28.5 \\
\downarrow 38.5^{*}\end{array}$ & $351 \pm 111$ & - & $\begin{array}{c}8.1 \%(3 / 37) \\
\downarrow 16.1 \%\end{array}$ & $\begin{array}{c}8.1 \%(3 / 37) \\
\downarrow 2.7 \%\end{array}$ & $\begin{array}{l}115.4 \pm 18.2 \mathrm{~d} \\
-2.2 \pm 2.8^{*}, \mathrm{e}\end{array}$ \\
\hline
\end{tabular}


Table 2. Cont.

\begin{tabular}{|c|c|c|c|c|c|c|c|c|c|c|c|c|c|}
\hline Year & Author & $\mathbf{N}$ & $\begin{array}{c}\text { F-UP } \\
\text { (Months) } \\
\text { (Range) }\end{array}$ & $\begin{array}{c}\text { Age (Years) } \\
\text { Mean } \\
\text { (Range) }\end{array}$ & $\begin{array}{c}\text { Type of } \\
\text { RP }\end{array}$ & NS & UI & $\begin{array}{c}\text { PVR (mL) } \\
\Delta \text { PVR }\end{array}$ & $\begin{array}{c}\text { Bcap. }(\mathrm{mL}) \\
\Delta \text { Bcap. }(\mathrm{mL})\end{array}$ & $\begin{array}{c}\mathrm{BC} \\
\left.\text { (mL/cm } \mathrm{m}_{2} \mathrm{O}\right) \\
\text { or/and IBC } \\
\Delta \mathrm{BC} ; \Delta \mathrm{IBC}\end{array}$ & $\begin{array}{c}\text { BOO } \\
\Delta \text { BOO }\end{array}$ & $\begin{array}{c}\text { DO } \\
\Delta \mathrm{DO}\end{array}$ & $\begin{array}{c}\mathrm{DU} \\
\Delta \mathrm{DU}\end{array}$ \\
\hline \multirow[t]{3}{*}{2018} & Huang & 48 & Pre-op. & $72.1 \pm 5.68$ & LRP & $\begin{array}{c}37.5 \% \\
(18 / 48)\end{array}$ & - & $31.3 \pm 63.8$ & $296.0 \pm 106.9$ & $65.3 \pm 75.9$ & - & - & - \\
\hline & & 31 & 1 & & & & $\begin{array}{c}93.8 \% \\
(45 / 48)\end{array}$ & $30.26^{\mathrm{a}}$ & $302.1^{\mathrm{a}}$ & $\begin{array}{l}65.4^{\mathrm{a}} \\
\uparrow 0.1\end{array}$ & - & - & - \\
\hline & & 28 & 3 & & & & $\begin{array}{c}66.7 \% \\
(32 / 48)\end{array}$ & - & - & - & - & - & - \\
\hline \multirow[t]{2}{*}{2019} & Iguchi & 75 & Pre-op. & $67.6^{\mathrm{a}}$ & RALP & $\begin{array}{c}44 \% \\
(33 / 75)\end{array}$ & $0 \%$ & - & $226.9^{a}$ & - & - & $25.3 \%(19 / 75)$ & - \\
\hline & & 75 & 3 & & & & $\begin{array}{c}33.3 \% \\
(25 / 48)\end{array}$ & - & $207.8^{\mathrm{a}}$ & - & - & $\begin{array}{c}33.3 \%(25 / 75) \\
\uparrow 8 \% \\
\text { N } 17.3 \%(13 / 75) \\
\text { R } 36.8 \%(7 / 19)\end{array}$ & - \\
\hline \multirow[t]{2}{*}{2020} & Zhou & 35 & Pre-op. & $63.4 \pm 8.1$ & RALP & $\begin{array}{c}100 \% \\
(35 / 35)\end{array}$ & $0 \%$ & - & $\begin{array}{c}385.3 \\
(351.3-410.2)\end{array}$ & - & - & - & - \\
\hline & & 35 & 6 & & & & $0 \%$ & - & $\begin{array}{c}370.2 \\
(330.1-395.4)\end{array}$ & - & - & - & - \\
\hline 2021 & & 64 & 1 & & & & - & - & - & - & - & - & N 37\% 24/64 \\
\hline \multirow[t]{2}{*}{2021} & Lee & 61 & Pre-op. & $\begin{array}{c}69.0 \\
(61.0-73.0)\end{array}$ & RALP & $\begin{array}{c}82 \% \\
(50 / 61)\end{array}$ & - & $\begin{array}{c}41.0 \\
(17.0-70.0) \\
\end{array}$ & $\begin{array}{c}306.0 \\
(248.0356 .0)\end{array}$ & $57.0(44.0-80.0)$ & $\begin{array}{c}36.0 \\
(28.5-53.3)^{\mathrm{c}} \\
\end{array}$ & $9.8 \%(6 / 61)$ & \\
\hline & & 61 & 4 & & & & $\begin{array}{c}18 \% \\
(11 / 61)\end{array}$ & $\begin{array}{c}22.5 \\
(10.0-56.0) \\
\downarrow 18.5+\end{array}$ & $\begin{array}{c}287.5 \\
(229.3-340.8)\end{array}$ & $\begin{array}{c}57.5(41.0-80.0) \\
\uparrow 0.5+\end{array}$ & $\begin{array}{c}28.5(15.0-40.0) \\
\text { c } \\
\downarrow 7.5^{*}\end{array}$ & $\begin{array}{c}5.0 \%(3 / 61) \\
\downarrow 4.8 \% *\end{array}$ & - \\
\hline
\end{tabular}

Abbreviations: $\mathrm{N}$ = number of patients included; Pre-op. = preoperative; Post-op. = Postoperative; ORP = open RP; LRP laparoscopic RP; RALP = robot assisted laparoscopic RP NS = nerve sparing surgery; UI = urinary incontinence; PVR = post-void residual; Bcap = bladder capacity; BC = bladder compliance; IBC = impaired bladder compliance, $\%$ (n/N); $\mathrm{BOO}=$ bladder outlet obstruction, $\%(\mathrm{n} / \mathrm{N}) ; \mathrm{DO}=$ detrusor overactivity, \% $(\mathrm{n} / \mathrm{N}) ; \mathrm{DU}=$ detrusor underactivity, \% (n/N). $\Delta=$ indicate change in listed parameter $(\mathrm{e} . \mathrm{g}$., change in rate of $\mathrm{BOO}=$ bladder outlet obstruction, $\%(\mathrm{n} / \mathrm{N}) ; \mathrm{DO}=$ detrusor overactivity, $\%(\mathrm{n} / \mathrm{N}) ; \mathrm{DU}=$ detrusor underactivity, \% $(\mathrm{n} / \mathrm{N}) . \Delta=$ indicate change in listed parameter $($ e.g., , change in rate of
$\mathrm{BOO})$; If not indicated otherwise states for absolute change. Statistical significance or lack of significancy was indicated always when available. Bold text refers to rates of $(\mathrm{N})$ de novo $\mathrm{BOO})$; If not indicated otherwise states for absolute change. Statistical significance or lack of significancy was indicated always when available. Bold text refers to rates of ( $\mathbf{N}$ ) de novo $\mathrm{IBC}, \mathrm{BOO}, \mathrm{OD}, \mathrm{DU}$ or rates of IBC, BOO, OD, DU resolved (R) after RP. If not indicated otherwise $\mathbf{R}$ states for relative change. $\mathbf{N}$ and $\mathbf{R}$ are relative to previous follow-up assessment.
$\S=$ mean $^{+}=$median $^{*}=p<0$. $\S=$ mean; $\ddagger=$ median; ${ }^{*}=p<0.05 ;+=$ not significant; ${ }^{\mathrm{a}}=$ weighted arithmetic mean; ${ }^{\mathrm{b}}=\%$ of patients with IBC; ${ }^{\mathrm{c}}=\mathrm{BOOI}\left(\right.$ bladder outlet obstruction index), mean (range); ${ }^{\mathrm{d}}=\mathrm{BC}$ 列 available for follow-up. 
Table 3. Characteristics of studies reporting DU.

\begin{tabular}{|c|c|c|c|c|}
\hline Author & $\begin{array}{l}\text { F-UP (Months) } \\
\text { (Range) }\end{array}$ & Criteria of DU & Coincidence & PROMs \\
\hline \multirow[t]{3}{*}{ Giannantoni } & Pre-op. & & $\begin{array}{l}\text { DU + BOO } 16.3 \%(8 / 49) \\
\text { DU + DO } 14.3 \%(7 / 49)\end{array}$ & - \\
\hline & 1 & \multirow{2}{*}{ Schafer nomogram } & $\begin{array}{c}\text { DU + DO 36.7\% (18/49); } \uparrow 22.3 \% \text { * } \\
\text { DU + ISD } 57.1 \%(28 / 49) *\end{array}$ & - \\
\hline & 8 & & $\begin{array}{l}\text { DU + DO } 22.4 \%(11 / 49) \dagger \ddagger \\
\text { DU + ISD 30.6\% }(15 / 49) * \ddagger\end{array}$ & - \\
\hline \multirow[t]{4}{*}{ Natsume } & Pre-op. & & 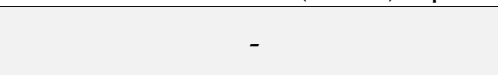 & $\begin{array}{l}\text { IPSS } 10.8 \pm 8.3(0-28) \\
\text { QOLsc } 3.3 \pm 1.9(0-6)\end{array}$ \\
\hline & 1 & \multirow{3}{*}{ Schafer nomogram } & - & $\begin{array}{l}\text { IPSS } 13.3 \pm 10.4 ; \uparrow 3,5^{*} ; \\
\text { QOLsc } 4.3 \pm 1.5 ; \uparrow 1^{*}\end{array}$ \\
\hline & 3 & & - & IPSS $9.5 \pm 9.5 ;$ QOLsc $2.9 \pm 2.1$ \\
\hline & 6 & & - & $\begin{array}{c}\text { IPSS } 6.1 \pm 6.4 \\
\text { QOLsc } 1.8 \pm 1.3^{*}\end{array}$ \\
\hline \multirow[t]{3}{*}{ Giannantoni } & Pre-op. & & $\mathrm{DO}+\mathrm{DU} 16.7 \%(9 / 54)$ & Strain voiders $20.4 \% 11 / 54$ \\
\hline & 8 & \multirow[b]{2}{*}{ Schafer nomogram } & $\begin{array}{c}\mathrm{DO}+\mathrm{DU} 38.9 \%(21 / 54) ; \uparrow 22,2 \% * \\
\text { N 29.6\% (16/54) } \\
\text { DU + ISD } 44.4 \%(24 / 54) *\end{array}$ & ( \\
\hline & 36 & & 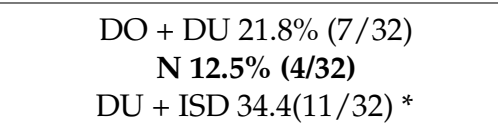 & - \\
\hline \multirow[t]{2}{*}{ Matsukawa } & Pre-op. & & - & - \\
\hline & $3.8 \S(2-5)$ & $\uparrow \mathrm{Pabd}$ and PdetQmax $<10 \mathrm{cmH}_{2} \mathrm{O}$ & $\mathrm{DO}+$ de novo DU $0 \%$ & - \\
\hline \multirow[t]{2}{*}{ Mitsui } & Pre-op. & & - & $\begin{array}{c}\text { IPSS } 8.6 \pm 7.0 ; \text { QOLsc } 3.4 \pm 1.5 \\
\text { DU (post-op) non-DU: } \\
\text { IPSS } 10.4 \pm 8.3 \text { vs. } 7.1 \pm 5.5 \dagger \\
\text { QOLsc } 3.9 \pm 1.5 \text { vs. } 2.9 \pm 1.4^{*}\end{array}$ \\
\hline & 12 & $\mathrm{WFmax}<10\left(\mathrm{~W} / \mathrm{m}^{2}\right)$ & - & $\begin{array}{c}\text { IPSS } 7.9 \pm 4.7+\text {; QOLsc } 2.5 \pm 1.6 ; \uparrow 0.9 \%{ }^{*} \\
\text { DU vs. non-DU: } \\
\text { IPSS } 8.6 \pm 4.2 \text { vs. } 7.2 \pm 5.2 \dagger \\
\text { QOLsc } 2.7 \pm 1.4 \text { vs. } 2.2 \pm 1.8 \dagger\end{array}$ \\
\hline \multirow[t]{2}{*}{ Dubelman } & Pre-op. & & - & - \\
\hline & 6.5 & WFmax $\leq 10\left(\mathrm{~W} / \mathrm{m}^{2}\right)$ & - & - \\
\hline
\end{tabular}


Table 3. Cont.

\begin{tabular}{|c|c|c|c|c|}
\hline Author & $\begin{array}{l}\text { F-UP (Months) } \\
\text { (Range) }\end{array}$ & Criteria of DU & Coincidence & PROMs \\
\hline \multirow[t]{2}{*}{ Mucciardi } & Pre-op. & & $\begin{array}{c}\text { DU + DO 6\% (6/100) } \\
\mathrm{DO}+\mathrm{DU}+\mathrm{IBC} 6 \%(6 / 100)\end{array}$ & - \\
\hline & 12 & $\begin{array}{c}\mathrm{BCI}<75, \mathrm{WFmax}<7 \mu \mathrm{W} / \mathrm{mm}^{2} \text { and } \\
\mathrm{MVDC}<7.5 \mathrm{~mm} / \mathrm{s} .\end{array}$ & $\begin{array}{c}\text { DU + DO 59.1\% (52/88); } \uparrow 53.1 \% \\
\text { N 52.3\% (46/8) } \\
\text { DO + DU + IBC 25\% (22/88); } 119 \% \\
\text { N 18.2\% (16/88) }\end{array}$ & - \\
\hline \multirow[t]{3}{*}{ Kadono } & Pre-op. & & $\begin{array}{c}\text { ISD + DU 0\%; DO + DU 0\% } \\
\text { DO + BOO } 16 \%(10 / 63)\end{array}$ & - \\
\hline & Post-op. & & $\begin{array}{c}\text { ISD + DU 60\%; }(38 / 63) ; \uparrow 60 \% ; \text { N 60\% (38/63) } \\
\text { DO + DU 6\% (4/63); } 6 \% ; \text { N 60\% (4/63) }\end{array}$ & - \\
\hline & 12 & Schafer nomogram & $\begin{array}{c}\text { ISD + DU 6\% (4/63); } \downarrow \text { 54\%; N } \mathbf{0 \%} \\
\text { DO + DU 5\% (3/63); } \downarrow 1 \% ; \mathbf{N ~ 0 \%} \\
\text { DO + BOO 2\% (1/63); N 0\% }\end{array}$ & - \\
\hline \multirow[t]{2}{*}{ Kitta } & Pre-op. & & - & IPSS $8.8 \pm 7.3 ;$ QOLsc score $3.5 \pm 1.5$ \\
\hline & 12 & $\begin{array}{c}\text { BCI; rV (Wmax), Line(W) and } \\
\text { W80-W20 }\end{array}$ & - & $\begin{array}{c}\text { IPSS } 8.1 \pm 4.8 ; \downarrow 0.8+ \\
\text { QOLsc score } 2.5 \pm 1.7 ; \downarrow 1 *\end{array}$ \\
\hline \multirow[t]{2}{*}{ Hata } & Pre-op. & & - & $\begin{array}{l}\text { Non-DU vs. DU (post-op) } \\
\text { IPSS } 7.3 \pm 5.7 \text { vs. } 11.7 \pm 8.3^{*} \\
\text { QOL } 2.6 \pm 1.5 \text { vs. } 3.7 \pm 1.5^{*} \\
\text { OABSS } 2.8 \pm 2.5 \text { vs. } 3.6 \pm 2.0 \dagger\end{array}$ \\
\hline & 1 & $\begin{aligned} \text { pdetQmax } & \leq 25 \mathrm{cmH}_{2} \mathrm{O} \text { and Qmax } \\
& \leq 15 \mathrm{~mL} / \mathrm{s}\end{aligned}$ & - & $\begin{array}{l}\text { Non-DU vs. DU (post-op) } \\
\text { IPSS } 11.3 \pm 6.9 \text { vs. } 16.7 \pm 9.0^{*} \\
\text { QOL } 3.5 \pm 1.9 \text { vs. } 4.4 \pm 1.6^{*} \\
\text { OABSS } 6.8 \pm 3.9 \text { vs. } 9.2 \pm 3.7 *\end{array}$ \\
\hline
\end{tabular}

Abbreviations: $\mathrm{N}$ = number of patients included; Pre-op = preoperative; Post-op = Postoperative; DU—detrusor underactivity, ISD = intrinsic sphincter deficiency; $\mathrm{PROMs}=$ patient-reported outcome measures $\mathrm{BCI}=$ bladder contractility index; $\mathrm{MVDC}=$ maximum velocity of detrusorial contraction; WFmax $=$ power at maximum flow; $\mathrm{QOL}=\mathrm{quality}$ of life score; OABSS = Overactive Bladder Symptom Score. ${ }^{*} p<0,05 ; \dagger=$ not significant; $\ddagger=$ compared to baseline; $\mathbf{N}=$ de novo, relative to previous follow-up assessment. 


\subsubsection{Detrusor Overactivity}

Detrusor overactivity was the most frequently reported outcome, being assessed in 23 studies (Table 2). The median rate of DO, preoperatively, was $25 \%$ (5\% to $76 \%$ ) in 18 studies [17-21,23,24,26,29-32,34-36,38,39,43]. The rate of de novo DO initially after RP was evaluated in 14 [18-20,23,24,26,29-32,34-36,39] studies, with the timing of the first follow-up ranging from a few days following catheter removal to 22 months. The median rate of de novo was $15 \%(2.3 \%$ to $54.4 \%)$. On the other hand, in ten studies [17,20,23,24,26,30-32,34,38], DO resolved initially in $19.6 \%$ to $87.5 \%$ of affected patients, with a median of 33\%. During further observation in four studies $[19,20,26,39]$, lasting from 6 to 36 months, DO occurred de novo in 1.4\% to 15.9\% (median of 9.5\%) of patients and resolved in $25 \%$ to $42.8 \%$ of them (median of $25.9 \%$ ).

Changes in absolute rates of DO on first follow-up evaluation differ substantially between 18 studies with a median of $-2,5 \%$ ( $-29.6 \%$ to $56.2 \%$ ) [17-21,23,24,26,29-32,34,35,37-39,43]. Rates of DO increased initially in seven studies with a median of $8 \%(6.4 \%$ to $56.2 \%$ ) $[19,20,23,31,33,35,39]$, but decreased in nine studies (median of $5.7 \%$, range from $2.7 \%$ to $29 \%$, $[17,18,24,26,29,30,34,38,43]$.

\subsubsection{Bladder Outlet Obstruction}

Bladder outlet resistance was described preoperatively solely as a continuous variable (bladder outlet obstruction index-BOOI) in three studies $[24,36,43]$ and as a rate of BOO in eight studies [18-20,26,29,31,34,39] (Table 2). Baseline rates of BOO varied between studies with a median of $27.8 \%$ (19\% to $59.3 \%)$. The most pronounced change after surgery was a decrease of absolute rates of BOO, with a median of $19.8 \%$ (4.7\% to 51.9\%) and resolution of $\mathrm{BOO}$ in $88 \%$ to $93,8 \%$ (median of $92 \%$ ) of affected patients (value relative to preoperative status) $[20,26,31]$. In minority of patients (median of $12 \%$, range from $3.2 \%$ to $15.9 \%$ ) BOO occurred de novo indicating usually formation of vesicourethral anastomosis stricture $[19,20,26,31,35,36]$.

\subsubsection{Bladder Compliance}

Bladder compliance was reported in 15 studies preoperatively [18-22,24-26,31,33-36,39,43] and in 12 [18-22,24-26,31,33,34,36,43] studies postoperatively (Table 2). In eight studies it was expressed as continues variable (mean compliance; $\mathrm{ml} / \mathrm{cmH} 2 \mathrm{O}$ ) whereas in six studies it was stated as rate of impaired contractility (IBC, compliance $<20 \mathrm{~mL} / \mathrm{cmH}_{2} \mathrm{O}$ ) or both. In four studies rates of IBC increased initially ranging with median of $17.5 \%$ $(6 \%$ to $40 \%)$ [18-20,26]. There was no initial decrease in rates of IBC reported, however it was reported during further observation (up to 36 months) in three studies with median of $25.6 \%(8.1 \%$ to $31.8 \%)[19,20,26]$. Rates of the novo IBC were reported in four studies with median of $13.3 \%(3.2 \%$ to $41.3 \%)$ [18-20,26], whereas resolve of IBC was reported with median of $4.8 \%(0 \%$ to $47 \%)[19,20,26,34]$.

\section{Discussion}

This systematic review revealed a high incidence of bladder dysfunction in men qualified for RP. The median rates of DU, DO, and BOO preoperatively were as high as $25 \%, 40.8 \%$, and $27.8 \%$, respectively. Additionally, RP substantially impacted LUT, which was expressed by both resolution and de novo occurrence of selected alterations in bladder function. All studies report an almost entirely homogenous decline in absolute rates of obstruction after RP. A much more complex picture pertains to DU and DO. De novo DU was reported with a high divergence of rates which varied from $9.1 \%$ to $37 \%$ across studies. On the other hand, preexisting DU resolved in $7 \%$ to $35.5 \%$ of studied populations. Similar changes, although with greater intensity, were revealed in terms of DO. Resolution of preoperative DO was reported with rates from $19.6 \%$ to $87.5 \%$ (median of $33 \%$ ). Additionally, in a substantial percentage of patients $(2.3 \%$ to $54.4 \%$ with a median of $15 \%$ ), the occurrence of de novo DO was found. Data corresponding to the influence 
of these alterations on patients' complaints and quality of life is, however, inconsistently reported across studies.

Men newly diagnosed with organ-confined PCa are often affected by LUTS. Although the preoperative incidence of LUTS was not a point of preplanned synthesis in this review, it may be indirectly illustrated by rates of LUT dysfunction on urodynamic study preoperatively. The coexistence of PCa and LUTS may be explained by the fact that incidences of both increase with age $[45,46]$, along with the phenomenon that patients with LUTS are more likely to be tested for PCa [47]. It has been previously shown that $12.1-56 \%$ of men undergoing radical prostatectomy (RP) have preoperative LUTS $[48,49]$. In a recent study by Walker et al., mild, moderate, and severe LUTS were reported by $50.7 \%, 39.4 \%$, and $9.9 \%$ of patients, respectively [6]. This may be attributed to bladder dysfunction as well as BOO from benign or, less frequently, malignant enlargement of the prostate [6,50]. Detrusor dysfunction such as DO and DU were reported in $17.4 \%$ and $14.8 \%$ of men, respectively, whereas BOO in $29.5 \%$ [6]. This systematic review revealed higher median rates of these alterations, which may be due to the selection of screened population limited to patients who had UDS both before and after the surgery, according to the predefined outcomes. There is no doubt that high rates of preoperative $\mathrm{DU}, \mathrm{DO}$, and $\mathrm{BOO}$ complicate reliable assessment of the impact on LUT function RP may have. Therefore, it seems reasonable to operate with the rates of de novo (or the resolution of) $\mathrm{DU}, \mathrm{DO}$, and $\mathrm{BOO}$ after RP as surrogates of surgical influence on bladder function.

A wide range of reported rates of de novo DU and DO may be partially explained by divergent definitions of distinctive alterations across studies. It was particularly pronounced in the case of DU, with six different formulas used in 10 studies (Table 3). Furthermore, none of these were validated in the population of men subjected to RP [51-54]. The authors believe that the optimal method to evaluate bladder contractility in men after RP-the population with usually extraordinarily low outflow resistance-is the assessment of maximum isometric detrusor pressure [55-57]. Another reason for the variety of reported rates may be the disparities in the time between surgery and the first urodynamic assessment, which range from 10 days to 12 months. Considering the ability of the lower urinary tract to restore its function after RP, this may be an important factor [58]. De novo DU after RP was reported in 8 of 10 studies assessing bladder contractility. This indicates the relevancy of mentioned phenomenon. It is believed to be related to autonomic nerve damage during surgical dissection $[19,20]$. This applies specifically to the dissection in the proximity of the bladder neck and the removal of the seminal vesicles [59-62]. Contrary, reinnervation may explain the restoration of detrusor function in time [58], which was also reported in reviewed studies $[19,20,26,29]$. Furthermore, nerve-sparing surgery may lead to preservation of some autonomic nerves too, whereas pelvic lymphadenectomy, performed in selected cases, may escalate pelvic plexus injury. Although the status of nerve-sparing was reported in some of the reviewed studies, correlation with the rate of DU was not assessed. Only Hata et al. performed a multivariate analysis of factors that may contribute to the DU. Preoperative IBC was the only measure established as a predicting factor for the development of postoperative DU [42]. In regards to postulated pathophysiology, many different nerve-sparing techniques have been created to improve functional outcomes [63]. Despite differences, they aim in reducing injury to neurovascular bundles due to respect of anatomical details and avoidance of traction. In a recently published study by Cochetti et al., a novel RALP technique meeting these goals was validated as safe and effective with good functional outcomes [3].

Detrusor underactivity is widely known to cause a number of severe clinical problems, including voiding difficulties, retention, and urinary tract infections [64,65]. However, its relevance in patients with substantially decreased outflow resistance after RP is under debate with contradictory results regarding symptoms and QOL (Table 3). In the previously mentioned study by Hata et al., better results in terms of IPPS total score and QOL were observed in men without DU when compared with those with de novo DU, one month after the surgery [42]. 
A much more homogeneous influence of RP on BOO was reported, with all reviewed studies suggesting improvement in absolute rates of obstruction. As mostly attributed to $\mathrm{BPH}$ in this population, $\mathrm{BOO}$ is resolved by removing the prostate during surgery. Furthermore, some improvement is observed $[29,34,36,39,48,49]$ in terms of LUTS, being likely related to $\mathrm{BOO}$ in older men [66]. This is claimed to be responsible for the net improvement in PROMs in some studies [36,39]. However, as in the noncancer population $[67,68]$, in the studied cohort, not all LUTS could be attributed to preoperative BOO. The persistence of DO was reported in up to 51.5\% [19]. This almost mirrors the data of BPH patients in whom DO resolution ranged from $57.1 \%$ to $83.3 \%$ after benign prostate surgery [69]. Changes after RP are, however, more complicated, with de novo DO ranging from $2.3 \%$ to $54.4 \%$ with a median of $15 \%$. This result could be biased by the wide range of time span of postoperative assessment and heterogeneity of baseline populations across analyzed studies. Furthermore, it could be explained by an underestimation of BOO postoperatively.

A wide range of changes in the rate of DO after RP may also be explained by the inclusion of the study by Muccardi et al., which showed the highest rise in the postoperative rate of DO (by 56.2\%) and the highest rate of de novo DO (54.4\%), with no resolution of it in previously affected patients [35]. After exclusion of this paper, the range of de novo $\mathrm{DO}$ in remaining studies is from $2.3 \%$ to $20.9 \%$, and the range of absolute changes in DO rate is from $-29.6 \%$ to $12 \%$. Outcomes regarding DO reported by Muccardi et al. may be explained by the high rate of vesicourethral anastomosis stricture (VAUS) (12\%). Although those patients were excluded from postoperative UDS, these outcomes may raise a concern about subclinical VAUS, which may contribute to DO.

De novo DO is generally attributed to the partial denervation and devascularization due to the bladder neck mobilization, as well as alteration of bladder geometry after the surgery [20,39].

To the best of our knowledge, only a few systematic reviews have been published so far. The one conducted by Porena et al. included 19 studies, with only eight embracing postoperative and preoperative urodynamic findings [5]. Moreover, it was published in 2007, and therefore it did not include 20 relevant studies published up to date. At the time of conducting this systematic review, another review concentrating on the related problem was published [70]. The specific focus of our research is the detrusor underactivity, whereas Yao et al. concern all alterations equally. Additionally, we were able to include 10 relevant studies $[17,20,23,25,32,34,40-43]$ missed by the other review. On the contrary, we omitted four studies included in the study of Yao et al.; two $[27,28]$ were excluded due to the substantial overlap with the third study [26], one as it contains patients after salvage radiotherapy [71], and one because of the lack of postoperative UDS report [72]. Despite qualitative and quantitative differences in included studies between the reviews, conclusions are corroborated by the two.

Although being the most up-to-date and comprehensive summary of RP impact on bladder function, this systematic review has some limitations. Above all, it is limited by the level of evidence and the heterogeneity of included studies. This applies to different approaches to the intervention (RP), range of follow-up, as well as definitions and tools used to assess alterations of bladder function. There is also a lack of consensus regarding DU definition in this particular group of patients. Moreover, there are no randomised studies assessing the impact of RP on bladder function. It would be, however, very difficult to conduct such a study. Finally, some of the studies that fulfilled eligibility criteria suffer from the lack of adequate outcomes reporting. Authors postulate the need for further evaluation of bladder function alterations after RP in prospective studies with both validated PROMs and UDS. Considering the transient character of many functional alterations after prostate surgery and the time needed for recovery, the optimal period for such evaluation seems to be no sooner than 6 to 12 months after RP $[58,73,74]$. Furthermore, choosing proper tools for the assessment of bladder contractility after RP is challenging since outlet resistance is considerably low after surgery. Evaluation of isovolumetric bladder contraction with a stop 
test seems to be the optimal method for this purpose. Moreover, the potential influence of nerve-sparing and lymphadenectomy during RP on bladder function should be assessed.

\section{Conclusions}

$\mathrm{BOO}, \mathrm{DO}$, and DU are frequently diagnosed in men qualified for RP. BOO is improved after RP in the majority of patients; however, there is still a substantial rate of patients with persistent or de novo DU as well as DO. Hence, the impact of RP on the lower urinary tract seems to be more complex than that resulting from just BOO improvement. Both DU and DO may impair functional outcomes [75] and the quality of life; however, there is no adequate data, especially including PROMs, to make a final statement. Further studies are thus required to define factors that may predict the risk of permanent LUT dysfunction after RP and the potential role of UDS in that process.

Supplementary Materials: The following supporting information can be downloaded at: https:// www.mdpi.com/article/10.3390/medicina58030381/s1, Table S1: Searching strategies for electronic databases; Table S2: Baseline characteristics of patients without follow-up.

Author Contributions: Conceptualization, M.O. and J.D.; methodology, M.O. and M.K.; screening and eligibility assessment, M.O., K.B. and M.K.; data extraction, M.O. and K.B.; formal analysis, M.O.; data curation, M.O.; writing-original draft preparation, M.O.; writing-review and editing, J.D.; supervision, J.D.; project administration, M.O. All authors have read and agreed to the published version of the manuscript.

Funding: This research received no external funding.

Institutional Review Board Statement: The study did not require ethical approval.

Informed Consent Statement: Not applicable.

Data Availability Statement: All the data are available from the corresponding author upon reasonable request.

Conflicts of Interest: The authors declare no conflict of interest.

\section{Abbreviations}

$\begin{array}{ll}\text { IBC } & \text { impaired bladder compliance, } \\ \text { BOO bladder outlet obstruction, } \\ \text { DO detrusor overactivity, } \\ \text { DU detrusor underactivity. }\end{array}$

\section{References}

1. Ferlay, J.; Soerjomataram, I.; Dikshit, R.; Eser, S.; Mathers, C.; Rebelo, M.; Parkin, D.M.; Forman, D.; Bray, F. Cancer incidence and mortality worldwide: Sources, methods and major patterns in GLOBOCAN 2012. Int. J. Cancer 2015, 136, E359-E386. [CrossRef] [PubMed]

2. Cochetti, G.; de Vermandois, J.A.R.; Maulà, V.; Giulietti, M.; Cecati, M.; Del Zingaro, M.; Cagnani, R.; Suvieri, C.; Paladini, A.; Mearini, E. Role of miRNAs in prostate cancer: Do we really know everything? Urol. Oncol. 2020, 38, 623-635. [CrossRef] [PubMed]

3. Cochetti, G.; Del Zingaro, M.; Ciarletti, S.; Paladini, A.; Felici, G.; Stivalini, D.; Cellini, V.; Mearini, E. New Evolution of Robotic Radical Prostatectomy: A Single Center Experience with PERUSIA Technique. Appl. Sci. 2021, 11, 1513. [CrossRef]

4. Smith, D.P.; King, M.T.; Egger, S.; Berry, M.P.; Stricker, P.D.; Cozzi, P.; Ward, J.; O'Connell, D.L.; Armstrong, B.K. Quality of life three years after diagnosis of localised prostate cancer: Population based cohort study. BMJ 2009, 339, b4817. [CrossRef]

5. Porena, M.; Mearini, E.; Mearini, L.; Vianello, A.; Giannantoni, A. Voiding dysfunction after radical retropubic prostatectomy: More than external urethral sphincter deficiency. Eur. Urol. 2007, 52, 38-45. [CrossRef]

6. Walker, N.F.; Canagasingham, A.; Van Diepen, D.; Pirpiris, A.; Tse, V.; Leslie, S.; Thanigasalam, R.; Chan, L. Lower Urinary Tract Functional Assessment of Men Undergoing Radical Prostatectomy: Correlation of Preoperative Clinical and Urodynamic Parameters. Int. Neurourol. J. 2021, 25, 157-163. [CrossRef]

7. Yao, H.H.; Crump, R.T.; Charbonneau, C.; Khan, A.; Barton, C.; Brotherhood, H.; Jiang, J.; Carlson, K.V.; Baverstock, R.J. Baseline patient reported outcomes data shows high prevalence of overactive bladder, sexual dysfunction, depression and anxiety in Canadian men with newly diagnosed localized prostate cancer. Transl. Urol. 2020, 9, 2046-2053. [CrossRef] 
8. Thiruchelvam, N.; Cruz, F.; Kirby, M.; Tubaro, A.; Chapple, C.R.; Sievert, K.D. A review of detrusor overactivity and the overactive bladder after radical prostate cancer treatment. BJU Int. 2015, 116, 853-861. [CrossRef]

9. Matsukawa, Y.; Yoshino, Y.; Fujita, T.; Funahashi, Y.; Majima, T.; Ishida, S.; Kato, M.; Gotoh, M. Daily urine loss immediately after urethral catheter removal may be an effective predictor of long-term urinary incontinence following robot-assisted laparoscopic radical prostatectomy. Int. J. Clin. Pract. 2021, 75, e13736. [CrossRef]

10. Groutz, A.; Blaivas, J.G.; Chaikin, D.C.; Weiss, J.P.; Verhaaren, M. The pathophysiology of post-radical prostatectomy incontinence: A clinical and video urodynamic study. J. Urol. 2000, 163, 1767-1770. [CrossRef]

11. Majima, T.; Matsukawa, Y.; Funahashi, Y.; Kato, M.; Sassa, N.; Gotoh, M. Urodynamic evaluation before and after to RARP to identify pre and intraoperative factors affecting postoperative continence. Neurourol. Urodyn. 2021, 40, 1147-1153. [CrossRef] [PubMed]

12. Hoyland, K.; Vasdev, N.; Abrof, A.; Boustead, G. Post-radical prostatectomy incontinence: Etiology and prevention. Rev. Urol. 2014, 16, 181-188. [PubMed]

13. Boni, A.; Cochetti, G.; Del Zingaro, M.; Paladini, A.; Turco, M.; de Vermandois, J.A.R.; Mearini, E. Uroflow stop test with electromyography: A novel index of urinary continence recovery after RARP. Int. Urol. Nephrol. 2019, 51, 609-615. [CrossRef] [PubMed]

14. Moher, D.; Shamseer, L.; Clarke, M.; Ghersi, D.; Liberati, A.; Petticrew, M.; Shekelle, P.; Stewart, L.A. Preferred reporting items for systematic review and meta-analysis protocols (PRISMA-P) 2015 statement. Syst. Rev. 2015, 4, 1. [CrossRef]

15. Tacconelli, E. Systematic reviews: CRD's guidance for undertaking reviews in health care. Lancet Infect. Dis. 2010, 10, 226. [CrossRef]

16. Ouzzani, M.; Hammady, H.; Fedorowicz, Z.; Elmagarmid, A. Rayyan-a web and mobile app for systematic reviews. Syst. Rev. 2016, 5, 210. [CrossRef]

17. Constantinou, C.E.; Freiha, F.S.; Siroky, M.B. Impact of radical prostatectomy on the characteristics of bladder and urethra. J. Urol. 1992, 148, 1215-1219. [CrossRef]

18. Dubbelman, Y.; Groen, J.; Wildhagen, M.; Rikken, B.; Bosch, R. Quantification of changes in detrusor function and pressure-flow parameters after radical prostatectomy: Relation to postoperative continence status and the impact of intensity of pelvic floor muscle exercises. Neurourol. Urodyn. 2012, 31, 637-641. [CrossRef]

19. Giannantoni, A.; Mearini, E.; Di Stasi, S.M.; Mearini, L.; Bini, V.; Pizzirusso, G.; Porena, M. Assessment of bladder and urethral sphincter function before and after radical retropubic prostatectomy. J. Urol. 2004, 171, 1563-1566. [CrossRef]

20. Giannantoni, A.; Mearini, E.; Zucchi, A.; Costantini, E.; Mearini, L.; Bini, V.; Porena, M. Bladder and urethral sphincter function after radical retropubic prostatectomy: A prospective long-term study. Eur. Urol. 2008, 54, 657-664. [CrossRef]

21. Hellström, P.; Lukkarinen, O.; Kontturi, M. Urodynamics in radical retropubic prostatectomy. Scand. J. Urol. Nephrol. 1989, 23 , 21-24. [CrossRef] [PubMed]

22. Huang, H.C.; Jiang, Y.H.; Lin, V.C.H.; Tsai, Y.C.; Kuo, H.C. Possible predictor of early recovery on urinary continence after laparoscopic radical prostatectomy-Bladder neck level and urodynamic parameters. J. Formos. Med. Assoc. 2019, 118, $237-243$. [CrossRef] [PubMed]

23. Iguchi, K.; Tanaka, T.; Minami, A.; Kuratsukuri, K.; Uchida, J.; Nakatani, T. Characteristics of urodynamic study parameters associated with intermediate-term continence after robot-assisted radical prostatectomy in elderly patients. Aging Male 2019, 23, 1039-1045. [CrossRef] [PubMed]

24. Jiang, Y.H.; Hong, Y.L.; Kuo, H.C. Urodynamic changes in patients with prostate cancer undergoing robotic-assisted radical prostatectomy: A comparison with laparoscopic radical prostatectomy. Urol. Sci. 2017, 28, 174-179. [CrossRef]

25. John, H.; Sullivan, M.P.; Bangerter, U.; Hauri, D.; Yalla, S.V. Effect of radical prostatectomy on sensory threshold and pressure transmission. J. Urol. 2000, 163, 1761-1766. [CrossRef]

26. Kadono, Y.; Ueno, S.; Iwamoto, D.; Takezawa, Y.; Nohara, T.; Izumi, K.; Mizokami, A.; Namiki, M. Chronological Urodynamic Evaluation of Changing Bladder and Urethral Functions After Robot-assisted Radical Prostatectomy. Urology 2015, 85, 1441-1447. [CrossRef]

27. Kadono, Y.; Ueno, S.; Kadomoto, S.; Iwamoto, H.; Takezawa, Y.; Nakashima, K.; Nohara, T.; Izumi, K.; Mizokami, A.; Gabata, T.; et al. Use of preoperative factors including urodynamic evaluations and nerve-sparing status for predicting urinary continence recovery after robot-assisted radical prostatectomy: Nerve-sparing technique contributes to the reduction of postprostatectomy incontinence. Neurourol. Urodyn. 2016, 35, 1034-1039.

28. Kadono, Y.; Ueno, S.; Yaegashi, H.; Ofude, M.; Izumi, K.; Maeda, Y.; Mizokami, A.; Miwa, S.; Miyagi, T.; Namiki, M. Urodynamic evaluation before and immediately after robot-assisted radical prostatectomy. Urology 2014, 84, 106-111. [CrossRef]

29. Kitta, T.; Kanno, Y.; Chiba, H.; Moriya, K.; Maruyama, S.; Abe, T.; Shinohara, N. Radical prostatectomy restores detrusor contraction pattern according to pressure flow parameters. Int. J. Urol. 2017, 24, 301-307. [CrossRef]

30. Kleinhans, B.; Gerharz, E.; Melekos, M.; Weingärtner, K.; Kälble, T.; Riedmiller, H. Changes of urodynamic findings after radical retropubic prostatectomy. Eur. Urol. 1999, 35, 217-221. [CrossRef]

31. Majoros, A.; Bach, D.; Keszthelyi, A.; Hamvas, A.; Romics, I. Urinary incontinence and voiding dysfunction after radical retropubic prostatectomy (prospective urodynamic study). Neurourol. Urodyn. 2006, 25, 2-7. [CrossRef] [PubMed]

32. Matsukawa, Y.; Hattori, R.; Komatsu, T.; Funahashi, Y.; Sassa, N.; Gotoh, M. De novo detrusor underactivity after laparoscopic radical prostatectomy. Int. J. Urol. 2010, 17, 643-648. [CrossRef] [PubMed] 
33. Matsukawa, Y.; Hattori, R.; Yoshikawa, Y.; Ono, Y.; Gotoh, M. Laparoscopic versus open radical prostatectomy: Urodynamic evaluation of vesicourethral function. Int. J. Urol. 2009, 16, 393-396. [CrossRef] [PubMed]

34. Mitsui, T.; Tanaka, H.; Harabayashi, T.; Moriya, K.; Maruyama, S.; Abe, T.; Sazawa, A.; Shinohara, N.; Nonomura, K. Changes in Urodynamics and Lower Urinary Tract Symptoms after Radical Prostatectomy: Implications of Preoperative Detrusor Contractility. LUTS Low. Urin. Tract Symptoms 2012, 4, 82-86. [CrossRef] [PubMed]

35. Mucciardi, G.; Galì, A.; Inferrera, A.; Di Benedetto, A.; Macchione, L.; Mucciardi, M.; Magno, C. Longitudinal observational cohort study about detrusor underactivity as a risk factor for bladder neck contracture after retropubic radical prostatectomy: Preliminary results. Int. Urol. Nephrol. 2013, 45, 721-726. [CrossRef] [PubMed]

36. Natsume, O.; Kondo, H.; Cho, M.; Fujimoto, K.; Ozono, S.; Hirao, Y. The impact of radical prostatectomy on patient well-being: A prospective urodynamic study focused on detrusor function. Hinyokika Kiyo 2004, 50, 1-6.

37. Noguchi, M.; Shimada, A.; Nakashima, O.; Kojiro, M.; Matsuoka, K. Urodynamic evaluation of a suspension technique for rapid recovery of continence after radical retropubic prostatectomy. Int. J. Urol. 2006, 13, 373-378. [CrossRef]

38. Rudy, D.C.; Woodside, J.R.; Crawford, E.D. Urodynamic evaluation of incontinence in patients undergoing modified Campbell radical retropubic prostatectomy: A prospective study. J. Urol. 1984, 132, 708-712. [CrossRef]

39. Song, C.; Lee, J.; Hong, J.H.; Choo, M.S.; Kim, C.S.; Ahn, H. Urodynamic interpretation of changing bladder function and voiding pattern after radical prostatectomy: A long-term follow-up. BJU Int. 2010, 106, 681-686. [CrossRef]

40. Zhou, X.; Fu, B.; Zhang, C.; Liu, W.; Guo, J.; Chen, L.; Lei, E.; Zhang, X.; Wang, G. Transvesical robot-assisted radical prostatectomy: Initial experience and surgical outcomes. BJU Int. 2020, 126, 300-308. [CrossRef]

41. Connolly, J.A.; Presti, J.C., Jr.; Carroll, P.R. Anterior bladder neck tube reconstruction at radical prostatectomy preserves functional urethral length a comparative urodynamic study. Br. J. Urol. 1995, 75, 766-770. [CrossRef] [PubMed]

42. Hata, J.; Onagi, A.; Tanji, R.; Honda-Takinami, R.; Matsuoka, K.; Hoshi, S.; Sato, Y.; Ogawa, S.; Kataoka, M.; Haga, N.; et al. Prevalence and predictors of postoperative detrusor underactivity after robot-assisted radical prostatectomy: A prospective observational study. Int. J. Urol. 2021, 28, 734-740. [CrossRef] [PubMed]

43. Lee, D.S.; Lee, S.J. Urodynamic evaluation of patients with localized prostate cancer before and 4 months after robotic radical prostatectomy. Sci. Rep. 2021, 11, 3632. [CrossRef]

44. Schäfer, W.; Abrams, P.; Liao, L.; Mattiasson, A.; Pesce, F.; Spangberg, A.; Sterling, A.M.; Zinner, N.R.; van Kerrebroeck, P. Good urodynamic practices: Uroflowmetry, filling cystometry, and pressure-flow studies. Neurourol. Urodyn. 2002, 21, 261-274. [CrossRef]

45. Bell, K.J.; Del Mar, C.; Wright, G.; Dickinson, J.; Glasziou, P. Prevalence of incidental prostate cancer: A systematic review of autopsy studies. Int. J. Cancer 2015, 137, 1749-1757. [CrossRef] [PubMed]

46. Irwin, D.E.; Milsom, I.; Hunskaar, S.; Reilly, K.; Kopp, Z.; Herschorn, S.; Coyne, K.; Kelleher, C.; Hampel, C.; Artibani, W.; et al. Population-based survey of urinary incontinence, overactive bladder, and other lower urinary tract symptoms in five countries: Results of the EPIC study. Eur. Urol. 2006, 50, 1306-1314. [CrossRef] [PubMed]

47. Weight, C.J.; Kim, S.P.; Jacobson, D.J.; McGree, M.E.; Boorjian, S.A.; Thompson, R.H.; Leibovich, B.C.; Karnes, R.J.; St Sauver, J. The effect of benign lower urinary tract symptoms on subsequent prostate cancer testing and diagnosis. Eur. Urol. 2013, 63, 1021-1027. [CrossRef]

48. Masters, J.G.; Rice, M.L. Improvement in urinary symptoms after radical prostatectomy: A prospective evaluation of flow rates and symptom scores. BJU Int. 2003, 91, 795-797. [CrossRef]

49. Schwartz, E.J.; Lepor, H. Radical retropubic prostatectomy reduces symptom scores and improves quality of life in men with moderate and severe lower urinary tract symptoms. J. Urol. 1999, 161, 1185-1188. [CrossRef]

50. Bhindi, A.; Bhindi, B.; Kulkarni, G.S.; Hamilton, R.J.; Toi, A.; van der Kwast, T.H.; Evans, A.; Zlotta, A.R.; Finelli, A.; Fleshner, N.E. Modern-day prostate cancer is not meaningfully associated with lower urinary tract symptoms: Analysis of a propensity score-matched cohort. Can. Urol. Assoc. J. 2017, 11, 41-46. [CrossRef]

51. Schäfer, W. Principles and clinical application of advanced urodynamic analysis of voiding function. Urol. Clin. N. Am. 1990, 17, 553-566. [CrossRef]

52. Griffiths, D.; van Mastrigt, R.; Bosch, R. Quantification of urethral resistance and bladder function during voiding, with special reference to the effects of prostate size reduction on urethral obstruction due to benign prostatic hyperplasia. Neurourol. Urodyn. 1989, 8, 29-52. [CrossRef]

53. van Mastrigt, R. Estimation of the maximum contraction velocity of the urinary bladder from pressure and flow throughout micturition. Urol. Res. 1990, 18, 149-154. [CrossRef] [PubMed]

54. Abrams, P. Bladder outlet obstruction index, bladder contractility index and bladder voiding efficiency: Three simple indices to define bladder voiding function. BJU Int. 1999, 84, 14-15. [CrossRef]

55. Sullivan, M.P.; DuBeau, C.E.; Resnick, N.M.; Cravalho, E.G.; Yalla, S.V. Continuous occlusion test to determine detrusor contractile performance. J. Urol. 1995, 154, 1834-1840. [CrossRef]

56. Griffiths, D. Detrusor contractility-order out of chaos. Scand. J. Urol. Nephrol. Suppl. 2004, 21, 93-100. [CrossRef]

57. Elliott, C.S.; Comiter, C.V. Maximum isometric detrusor pressure to measure bladder strength in men with postprostatectomy incontinence. Urology 2012, 80, 1111-1115. [CrossRef]

58. Chao, R.; Mayo, M.E. Incontinence after radical prostatectomy: Detrusor or sphincter causes. J. Urol. 1995, 154, 16-18. [CrossRef] 
59. Takenaka, A.; Soga, H.; Murakami, G.; Niikura, H.; Tatsumi, H.; Yaegashi, N.; Tanaka, K.; Fujisawa, M. Understanding anatomy of "hilus" of detrusor nerves to avoid bladder dysfunction after pelvic surgery: Demonstration using fetal and adult cadavers. Urology 2009, 73, 251-257. [CrossRef]

60. Martínez Portillo, F.J.; Seif, C.; Braun, P.M.; Böhler, G.; Osmonov, D.K.; Leissner, J.; Hohenfellner, R.; Alken, P.; Juenemann, K.P. Risk of detrusor denervation in antireflux surgery demonstrated in a neurophysiological animal model. J. Urol. 2003, 170, 570-573, discussion 573-574. [CrossRef]

61. Hollabaugh, R.S., Jr.; Dmochowski, R.R.; Kneib, T.G.; Steiner, M.S. Preservation of putative continence nerves during radical retropubic prostatectomy leads to more rapid return of urinary continence. Urology 1998, 51, 960-967. [CrossRef]

62. Spradling, K.; Khoyilar, C.; Abedi, G.; Okhunov, Z.; Wikenheiser, J.; Yoon, R.; Huang, J.; Youssef, R.F.; Ghoniem, G.; Landman, J. Redefining the Autonomic Nerve Distribution of the Bladder Using 3-Dimensional Image Reconstruction. J. Urol. 2015, 194, 1661-1667. [CrossRef] [PubMed]

63. Tavukçu, H.H.; Aytac, O.; Atug, F. Nerve-sparing techniques and results in robot-assisted radical prostatectomy. Investig. Clin. Urol. 2016, 57, S172-S184. [CrossRef] [PubMed]

64. Taylor, J.A., 3rd; Kuchel, G.A. Detrusor underactivity: Clinical features and pathogenesis of an underdiagnosed geriatric condition. J. Am. Geriatr. Soc. 2006, 54, 1920-1932. [CrossRef] [PubMed]

65. Drake, M.J.; Williams, J.; Bijos, D.A. Voiding dysfunction due to detrusor underactivity: An overview. Nat. Rev. Urol. 2014, 11, 454-464. [CrossRef]

66. Kupelian, V.; Wei, J.T.; O’Leary, M.P.; Kusek, J.W.; Litman, H.J.; Link, C.L.; McKinlay, J.B. Prevalence of lower urinary tract symptoms and effect on quality of life in a racially and ethnically diverse random sample: The Boston Area Community Health (BACH) Survey. Arch. Intern. Med. 2006, 166, 2381-2387. [CrossRef]

67. Chapple, C.R.; Wein, A.J.; Abrams, P.; Dmochowski, R.R.; Giuliano, F.; Kaplan, S.A.; McVary, K.T.; Roehrborn, C.G. Lower urinary tract symptoms revisited: A broader clinical perspective. Eur. Urol. 2008, 54, 563-569. [CrossRef]

68. Chapple, C.; Abrams, P.; Société Internationale d'Urologie (SIU). Lower Urinary Tract Symptoms (LUTS): An International Consultation on Male LUTS; SIU: Montreal, QC, Canada, 2012.

69. Creta, M.; Longo, N.; Mangiapia, F.; Arcaniolo, D.; Imbimbo, C.; Mirone, V.; Fusco, F. Detrusor overactivity and underactivity: Implication for lower urinary tract symptoms related to benign prostate hyperplasia diagnosis and treatment. Minerva Urol. Nephrol. 2021, 73, 59-71. [CrossRef]

70. Yao, H.H.; Hoe, V.; Crump, R.T.; Sengupta, S.; O'Connell, H.E.; Carlson, K.V.; Baverstock, R.J. Impact of radical prostatectomy on bladder function as demonstrated on urodynamics study-A systematic review. Neurourol. Urodyn. 2021, 40, 582-603. [CrossRef]

71. Ervandian, M.; Djurhuus, J.C.; Høyer, M.; Graugaard-Jensen, C.; Borre, M. Long-term urodynamic findings following radical prostatectomy and salvage radiotherapy. Scand. J. Urol. 2018, 52, 20-26. [CrossRef]

72. Aboseif, S.R.; Konety, B.; Schmidt, R.A.; Goldfien, S.H.; Tanagho, E.A.; Narayan, P.A. Preoperative urodynamic evaluation: Does it predict the degree of urinary continence after radical retropubic prostatectomy? Urol. Int. 1994, 53, 68-73. [CrossRef] [PubMed]

73. Choi, H.; Kim, J.H.; Shim, J.S.; Park, J.Y.; Kang, S.H.; Moon du, G.; Cheon, J.; Lee, J.G.; Kim, J.J.; Bae, J.H. Prediction of persistent storage symptoms after transurethral resection of the prostate in patients with benign prostatic enlargement. Urol. Int. 2014, 93, 425-430. [CrossRef] [PubMed]

74. Antunes, A.A.; Iscaife, A.; Reis, S.T.; Albertini, A.; Nunes, M.A.; Lucon, A.M.; Nahas, W.C.; Srougi, M. Can we predict which patients will experience resolution of detrusor overactivity after transurethral resection of the prostate? J. Urol. 2015, 193, 2028-2032. [CrossRef] [PubMed]

75. Pastore, A.L.; Palleschi, G.; Illiano, E.; Zucchi, A.; Carbone, A.; Costantini, E. The role of detrusor overactivity in urinary incontinence after radical prostatectomy: A systematic review. Minerva Urol. Nefrol. 2017, 69, 234-241. [CrossRef] [PubMed] 